\title{
Universiteit
}

Leiden

The Netherlands

\section{Method to determine defect positions below a metal surface by STM}

Avotina, Y.S.; Kolesnichenko, Y.A.; Omelyanchouk, A.N.; Otte, A.F.; Ruitenbeek, J.M. van

\section{Citation}

Avotina, Y. S., Kolesnichenko, Y. A., Omelyanchouk, A. N., Otte, A. F., \& Ruitenbeek, J. M. van. (2005). Method to determine defect positions below a metal surface by STM. Physical Review $B, 71,115430$. doi:10.1103/PhysRevB.71.115430

Version: $\quad$ Not Applicable (or Unknown)

License: $\quad$ Leiden University Non-exclusive license

Downloaded from: https://hdl.handle.net/1887/62281

Note: To cite this publication please use the final published version (if applicable). 


\title{
Method to determine defect positions below a metal surface by STM
}

\author{
Ye. S. Avotina, ${ }^{1,2}$ Yu. A. Kolesnichenko, ${ }^{1}$ A. N. Omelyanchouk, ${ }^{1}$ A. F. Otte, ${ }^{2}$ and J. M. van Ruitenbeek ${ }^{2}$ \\ ${ }^{1}$ B.I. Verkin Institute for Low Temperature Physics and Engineering, National Academy of Sciences of Ukraine, 47, Lenin Ave., 61103, \\ Kharkov, Ukraine \\ ${ }^{2}$ Kamerlingh Onnes Laboratorium, Universiteit Leiden, Postbus 9504, 2300 Leiden, The Netherlands
}

(Received 24 September 2004; published 29 March 2005)

\begin{abstract}
The oscillatory voltage dependence of the conductance of a quantum point contact in the presence of a single pointlike defect has been analyzed theoretically. Such signals are detectable and may be exploited to obtain information on defect positions below a metal surface. Both tunnel junctions and ballistic contacts of adiabatic shape have been considered. The effect of quantum interference has been taken into account between the principal wave that is directly transmitted through the contact and the partial wave that is scattered by the contact and the defect. This effect leads to oscillations of the conductance as a function of applied voltage. We obtain the dependence of the period and amplitude of the conductance oscillations on the position of the defect inside the metal.
\end{abstract}

DOI: $10.1103 /$ PhysRevB.71.115430

PACS number(s): 73.23.-b, 72.10.Fk

\section{INTRODUCTION}

In the two decades following its invention, scanning tunneling microscopy (STM) has proved to be a valuable tool for investigating surfaces on an atomic scale. More recently, several experiments show a growing interest in the study of structures that are situated in the bulk below the surface in both semiconductors and metals. Whereas in the former (i.e., semiconductor) case the absence of effective screening allows dopants down to the third subsurface layer to be viewed directly as apparent topographic features, ${ }^{1}$ the situation for metals turns out to be somewhat more complicated. One method that has been suggested for imaging structures buried in metal involves several surface study techniques to be employed simultaneously in combination with STM. ${ }^{2}$ However, although this experiment has lead to successful identification of subsurface defects, it cannot be used as a tool for probing the exact depth. Also, its employability is limited to certain specific alloys only. A more successful approach, however, seems to be by probing standing electron waves. ${ }^{3,4}$ The groundwork of these experiments is as described in Ref. 5, where $\mathrm{Cu}(111)$ surface states form a two-dimensional nearly free electron gas. When scattered from step edges or adatoms, these states then form standing waves that can be probed by scanning tunneling spectroscopy (STS).

Although it has been proposed to utilize these surface states for imaging subsurface impurities, ${ }^{6}$ the exponential decay of the wave-function amplitudes into the bulk will limit the effective range to the topmost layers only. Bulk states, however, of which the square falls off with only $r^{2}$, form a good alternative. To demonstrate this, we mention results that were obtained by bulk state spectroscopy on relatively large structures, such as Ar bubbles submerged in Al (Ref. 3) and $\mathrm{Si}(111)$ step edges buried under a thin film of $\mathrm{Pb}^{4}$ In these experiments, bulk electrons are found to be confined in a vertical quantum well between the surface and the top plane of the object of interest.

In this paper we show that the investigation of the nonlinear conductance of a point contact placed on a metal surface makes it possible to determine the position of pointlike defects, such as the vacancies or foreign atoms inside the metal in the vicinity of the contact. We consider theoretical models both for the cases of a tunnel point contact and for a ballistic quantum contact. We look for conductance oscillations caused by interference of electrons that are transmitted directly, and electrons that are first backscattered elastically by the defect and again scattered forward by the contact (i.e., the tip-sample junction), much in the same way as was described for atomic point contacts in Refs. 7-9. The effect of such quantum interference on the nonlinear conductance of quantum wires was theoretically analyzed in Refs. 11-13, but the point-contact geometry was not yet studied.

The organization of this paper is as follows. In Sec. II we consider a tunnel junction in the limit of a high potential barrier. The interaction of the transmitted electrons with a single impurity near the junction is taken into account by perturbation theory with the electron-impurity interaction as the small parameter. A general analytical expression for the voltage dependence of conductance $G(V)$ is obtained. It defines $G(V)$ in terms of the contact diameter, the distance between contact and the impurity and the parameters that characterize the metal, and the transmission of the tunnel junction. In Sec. III the conductance of a ballistic quantum contact of adiabatic shape is analyzed. In absence of a barrier inside the contact, electrons can still be reflected from it due to the variation of the confining potential. The influence on electron scattering by an explicit barrier potential in the center of the contact is also discussed. As in Sec. II, assuming the electron-impurity interaction to be small, we derive an expression for $G(V)$ and its dependence on the position of the defect. In Sec. IV we conclude by discussing the possibilities for experimental exploitation of the conductance fluctuations for subsurface imaging as well as the technical difficulties involved.

\section{TUNNEL POINT CONTACT}

Let us consider as a first model of our system a nontransparent interface located at $z=0$ between two metal half- 


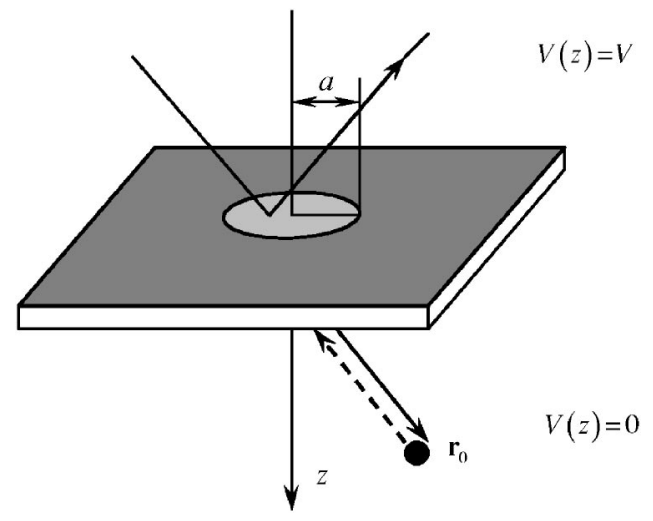

FIG. 1. Model of a tunnel junction contact as an orifice in an interface that is nontransparent for electrons except for a circular hole, where tunneling is allowed. Trajectories are shown schematically for electrons that are reflected from or transmitted through the contact and then reflected from a defect.

spaces in which there is an orifice (contact), as illustrated in Fig. 1. The potential barrier in the plane $z=0$ is taken to be a $\delta$ function

$$
U(\mathbf{r})=U f(\rho) \delta(z)
$$

where $\rho=(x, y)$ is a two-dimensional vector. The function $f(\rho) \rightarrow \infty$ in all points of the plane $z=0$ except in the contact, where $f(\rho)=1$. At a point $\mathbf{r}=\mathbf{r}_{0}$ in vicinity of the interface, in the half-space $z>0$, a pointlike defect is placed (see Fig. 1). The electron interaction with the defect is described by the potential

$$
g(\mathbf{r})=g \delta\left(\mathbf{r}-\mathbf{r}_{0}\right)
$$

where $g$ is the constant of the electron-impurity interaction. In this section we consider tunnel junctions and assume that the transmission probability of electrons through the orifice is small. In that case the applied voltage drops entirely over the barrier and we choose the electric potential as a step function $V(z)=V \Theta(-z)$ and take $e V>0$.

The electrical current $I(V)$ can be evaluated ${ }^{10}$ from the electron wave functions of the system $\psi_{\mathbf{k}}$,

$$
\begin{aligned}
I(V)= & \frac{e \hbar}{4 \pi^{3} m^{*}} \int d \mathbf{k} \int d \mathbf{S} \operatorname{Im}\left(\psi_{\mathbf{k}}^{*} \nabla \psi_{\mathbf{k}}\right) \Theta\left(k_{z}\right)\left[n_{F}\left(\varepsilon_{\mathbf{k}}\right)-n_{F}\left(\varepsilon_{\mathbf{k}}\right.\right. \\
& +e V)] .
\end{aligned}
$$

Here, $\varepsilon_{\mathbf{k}}=\hbar^{2} \mathbf{k}^{2} / 2 m^{*}$ is the electron energy, $\mathbf{k}$ is the electron wave vector, $m^{*}$ is an effective mass of electron, and $n_{F}\left(\varepsilon_{\mathbf{k}}\right)$ is the Fermi distribution function. The real-space integration is performed over a surface overlapping the contacts in the region $z>0$. At low temperatures the tunnel current is due to those electrons in the half-space $z<0$ having an energy between the Fermi energy $\varepsilon_{F}$ and $\varepsilon_{F}+e V$ because, on the other side of the barrier, only states with $\varepsilon_{\mathbf{k}} \geqslant \varepsilon_{F}$ are available.

The wave function $\psi_{\mathbf{k}}$ satisfies the Schrödinger equation

$$
\nabla^{2} \psi_{\mathbf{k}}(\rho, z)+\frac{2 m^{*}}{\hbar^{2}}\left[\varepsilon_{\mathbf{k}}-U(\mathbf{r})-g(\mathbf{r})-e V(z)\right] \psi_{\mathbf{k}}(\rho, z)=0
$$

where the wave vector $\mathbf{k}=\left(\varkappa, k_{z}\right)$ has components $\varkappa$ and $k_{z}$ parallel and perpendicular to interface, respectively. As shown in Ref. 14, Eq. (4) can be solved for arbitrary form of the function $f(\rho)$ in the limit $1 / U \rightarrow 0$. The wave function $\psi_{\mathbf{k}}(\rho, z)$ for $k_{z}>0$ in the main approximation of the small parameter $\sim 1 / U$ takes the form

$$
\begin{gathered}
\psi_{\mathbf{k}}(\rho, z)=e^{i \varkappa \rho}\left(e^{i \tilde{k} z}-e^{-i \tilde{k} z}\right)+\frac{1}{U} \varphi_{\tilde{\mathbf{k}}}^{(-)}(\rho, z) \quad(z<0), \\
\psi_{\mathbf{k}}(\rho, z)=\frac{1}{U} \varphi_{\mathbf{k}}^{(+)}(\rho, z) \quad(z>0),
\end{gathered}
$$

where $\tilde{\mathbf{k}}=(\varkappa,-\tilde{k}), \tilde{k}=\sqrt{k_{z}^{2}+2 m e V / \hbar^{2}}$. The function $\psi_{\mathbf{k}}(\rho, z)$ satisfies the conditions of continuity and the condition of the jump of its derivative at the boundary $z=0$. At large $U$ these conditions are reduced to

$$
\begin{aligned}
& \varphi_{\mathbf{k}}^{(-)}(\rho, 0)=\varphi_{\mathbf{k}}^{(+)}(\rho, 0), \\
& i \tilde{k}=\frac{m^{*}}{\hbar^{2}} f(\rho) \varphi_{\mathbf{k}}^{(+)}(\rho, 0) .
\end{aligned}
$$

In the absence of the defect $(g=0)$ the wave function was obtained in Ref. 14,

$$
\varphi_{0 \mathbf{k}}^{(+)}(\rho, z)=-\frac{i \hbar^{2} \tilde{k}}{2 \pi m^{*}} \int_{-\infty}^{\infty} d \varkappa^{\prime} F\left(\varkappa-\varkappa^{\prime}\right) e^{i \varkappa^{\prime} \rho+i k_{z}^{\prime} z}
$$

where

$$
F\left(\varkappa-\varkappa^{\prime}\right)=\int_{-\infty}^{\infty} d \rho \frac{e^{i\left(\varkappa-\varkappa^{\prime}\right) \rho}}{f(\rho)}
$$

and $k_{z}^{\prime}=\sqrt{\varkappa^{2}+k_{z}^{2}-\varkappa^{\prime 2}}$. For a circular contact of a radius $a$, defined by $f(|\rho| \leqslant a)=1$ and $f(|\rho|>a) \rightarrow \infty$, the function $F\left(x-x^{\prime}\right)$ takes the form

$$
F\left(\varkappa-\varkappa^{\prime}\right)=\frac{2 a J_{1}\left(\left|x-\varkappa^{\prime}\right| a\right)}{\left|\varkappa-\varkappa^{\prime}\right|} .
$$

In order to introduce the effect of the impurity we solve the Schrödinger equation for the Fourier components $\Phi_{\mathbf{k}}(\varkappa, z)$ of the function $\varphi_{\mathbf{k}}^{(+)}(\rho, z)$

$$
\varphi_{\mathbf{k}}^{(+)}(\rho, z)=\int_{-\infty}^{\infty} d \varkappa e^{i \varkappa \rho} \Phi_{\mathbf{k}}(\varkappa, z) .
$$

For $z>0$ this equation takes the form 


$$
\begin{aligned}
-\varkappa^{\prime 2} & \Phi_{\mathbf{k}}\left(\varkappa^{\prime}, z\right)+\frac{\partial^{2} \Phi_{\mathbf{k}}\left(\varkappa^{\prime}, z\right)}{\partial z^{2}} \\
& +\frac{2 m^{*}}{\hbar^{2}}\left[\varepsilon_{\mathbf{k}} \Phi_{\mathbf{k}}\left(\varkappa^{\prime}, z\right)-g \delta\left(z-z_{0}\right) e^{-i \varkappa^{\prime} \rho_{0}} \varphi_{\mathbf{k}}^{(+)}\left(\rho_{0}, z_{0}\right)\right]=0 .
\end{aligned}
$$

Integrating Eq. (13) near the point $z=z_{0}$, we obtain the effective boundary condition

$$
\begin{aligned}
\frac{\partial}{\partial z} \Phi_{\mathbf{k}}\left(\varkappa^{\prime}, z\right. & \left.=z_{0}+0\right)-\frac{\partial}{\partial z} \Phi_{\mathbf{k}}\left(\varkappa^{\prime}, z=z_{0}-0\right) \\
& =\frac{2 m^{*} g}{\hbar^{2}} e^{-i \varkappa^{\prime} \rho_{0}} \varphi_{\mathbf{k}}^{(+)}\left(\rho_{0}, z_{0}\right) .
\end{aligned}
$$

To proceed with further calculations we assume that the electron-impurity interaction constant $g$ is small and use perturbation theory. In this approximation we replace $\varphi_{\mathbf{k}}^{(+)}$by $\varphi_{0 \mathbf{k}}^{(+)}$(9) on the right-hand side of Eq. (14). Solving the Schrödinger equation (13) with the boundary conditions (7), (8), (14), and the condition of continuity of the function $\Phi_{\mathbf{k}}(\varkappa, z)$ at $z=z_{0}$, we obtain in the region $z>z_{0}$

$$
\Phi_{\mathbf{k}}\left(\varkappa^{\prime}, z\right)=t_{\mathbf{k}}\left(\varkappa^{\prime}\right) e^{i k_{z}^{\prime} z}
$$

where

$t_{\mathbf{k}}\left(\varkappa^{\prime}\right)=-\frac{i \hbar^{2} \widetilde{k}}{2 \pi m^{*}} F\left(\varkappa-\varkappa^{\prime}\right)-\frac{2 m^{*} g}{k_{z}^{\prime} \hbar^{2}} \varphi_{0 \mathbf{k}}^{(+)}\left(\rho_{0}, z_{0}\right) e^{-i \varkappa^{\prime} \rho_{0}} \sin \left(k_{z}^{\prime} z_{0}\right)$

Using Eq. (13) we find the wave function

$$
\begin{aligned}
& \varphi_{\mathbf{k}}^{(+)}(\rho, z)=\varphi_{0 \mathbf{k}}^{(+)}(\rho, z)-\frac{4 \pi m^{*} g}{\hbar^{2}} \varphi_{0 \mathbf{k}}^{(+)}\left(\rho_{0}, z_{0}\right)
\end{aligned}
$$

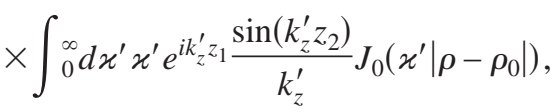

where $z_{1}=z, z_{2}=z_{0}$, when $z>z_{0}$, and $z_{1}=z_{0}, z_{2}=z$ when $0<z<z_{0}$. The modulus of the wave function (17) in a plane through the impurity and normal to the circular contact is illustrated in Fig. 2, for an incident wave vector normal to the interface $\left(k_{z}=k_{F}=(1 / \hbar) \sqrt{2 m^{*} \varepsilon_{F}}\right)$. One recognizes an interference pattern of partial waves reflected at the impurity with those emanating from the contact. These contain the information that we hope to extract.

Substituting wave function (6) into Eq. (3) and taking into account Eq. (17), we calculate the current-voltage characteristics $I(V)$. After integration over all directions of the wave vector $\mathbf{k}$ and integration over the space coordinate $\rho$ in a plane $z=\operatorname{const}\left(z>z_{0}\right)$, retaining only terms to first order in $g$ (i.e., ignoring multiple scattering at the impurity site), the current is given by

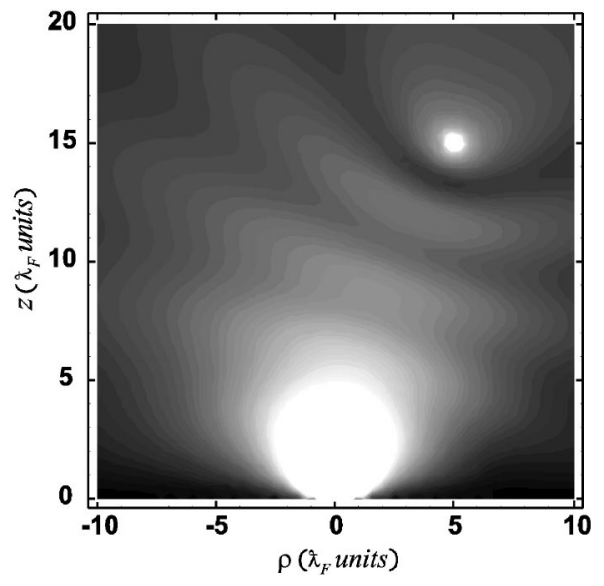

FIG. 2. Modulus of the wave function in the vicinity of a tunneling point contact in a plane perpendicular to the contact axis, having an impurity at $\left(\rho_{0}=5, z_{0}=15\right)$. The incident wave has a wave vector normal to the point contact $a=\lambda_{F} / 4 \pi$.

$$
\begin{aligned}
I(V)= & \frac{2 e \hbar^{5}}{2 \pi m^{*} U^{2}} \int_{0}^{\infty} d k k^{3} \iint \frac{d \rho_{1} d \rho_{2}}{f\left(\rho_{1}\right) f\left(\rho_{2}\right)}\left[n_{F}\left(\varepsilon_{\mathbf{k}}\right)-n_{F}\left(\varepsilon_{\mathbf{k}}\right.\right. \\
& +e V)] \times\left[\frac{A^{2}(k \rho)}{\rho^{4}}-2 \pi \frac{m^{*} g k}{\hbar^{2}} \frac{A\left(k \lambda_{1}\right) A\left(k \lambda_{2}\right)}{\lambda_{2}^{2} \lambda_{1}^{2}} z_{0}^{2}\left(\frac{A(k \rho)}{\rho^{2}}\right.\right. \\
& \left.\left.+\frac{2 m^{*} e V}{\hbar^{2}} \frac{\sin (k \rho)}{k \rho}\right)\right],
\end{aligned}
$$

where $\rho=\left|\rho_{1}-\rho_{2}\right|, \lambda_{1}=\sqrt{z_{0}^{2}+\left|\rho_{0}-\rho_{1}\right|^{2}}, \lambda_{2}=\sqrt{z_{0}^{2}+\left|\rho_{0}-\rho_{2}\right|^{2}}$, and

$$
A(x)=\frac{\sin x}{x}-\cos x
$$

Differentiating Eq. (18) with voltage $V$ and integrating over the absolute value $k$ of the wave vector, in the limit of low temperatures, $T=0$, we obtain the conductance $G(V)$ of the system

$$
\begin{aligned}
G(V)= & \frac{e^{2} \widetilde{k}_{F}^{2} \hbar^{3}}{\pi\left(m^{*} U\right)^{2}} \iint \frac{d \rho_{1} d \rho_{2}}{f\left(\rho_{1}\right) f\left(\rho_{2}\right)} \\
& \times\left[\frac{A^{2}\left(\tilde{k}_{F} \rho\right)}{\rho^{4}}-2 \pi^{*} \frac{m^{*} g \tilde{k}_{F}}{\hbar^{2}} \frac{A\left(\tilde{k}_{F} \lambda_{1}\right) A\left(\tilde{k}_{F} \lambda_{2}\right)}{\lambda_{1}^{2} \lambda_{2}^{2}} z_{0}^{2}\right. \\
& \times\left(\frac{A\left(\tilde{k}_{F} \rho\right)}{\rho^{2}}+\frac{2 m^{*} e V}{\hbar^{2}} \frac{\sin \left(\tilde{k}_{F} \rho\right)}{\tilde{k}_{F} \rho}\right) \\
& \left.-\frac{8 \pi}{\tilde{k}_{F}^{2}} \frac{m^{*} g}{\hbar^{2}} z_{0}^{2} \int_{k_{F}} \tilde{k}_{F} k^{3} d k \frac{\sin (k \rho)}{\rho} \frac{A\left(k \lambda_{1}\right) A\left(k \lambda_{2}\right)}{\lambda_{1}^{2} \lambda_{2}^{2}}\right],
\end{aligned}
$$

where $\tilde{k}_{F}=\sqrt{k_{F}^{2}+2 m^{*} e V / \hbar^{2}}$ is the Fermi wave vector accelerated by the potential difference, and we have assumed $e V / \varepsilon_{F}<1$. For $\lambda_{i}$ and $\rho$ much larger than the Fermi wave length $\lambda_{F}=1 / k_{F}$, the function $A$ oscillates with $\left(e V / \varepsilon_{F}\right) k_{F} \lambda_{i}$ and $\left(e V / \varepsilon_{F}\right) k_{F} \rho$, which results in a fluctuation of the conductance with applied voltage. The first term in square brackets 


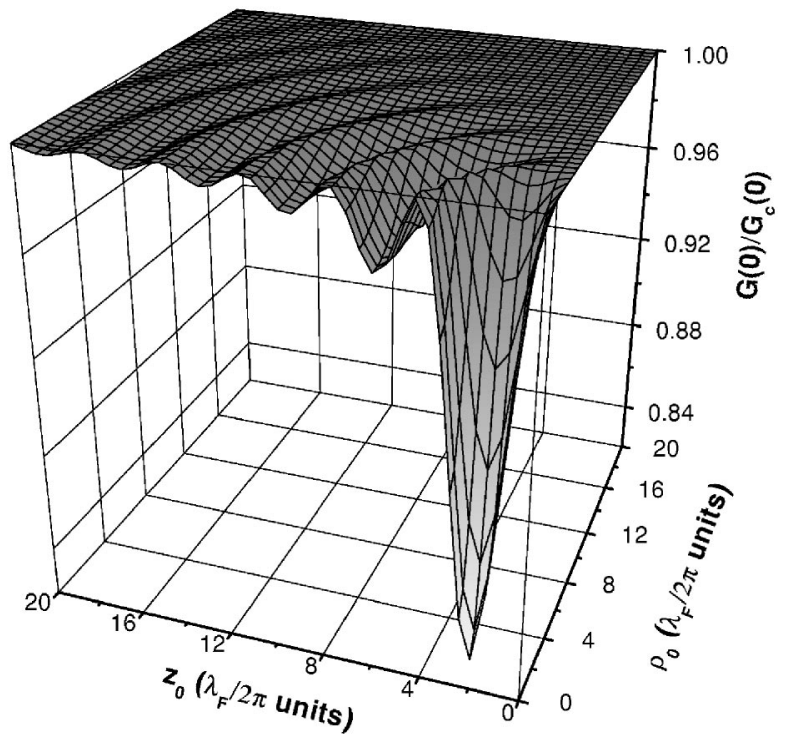

FIG. 3. Dependence of the normalized conductance $G / G_{c}$ for a single tunnel point contact as a function of the position of the defect $\left(\rho_{0}, z_{0}\right)$; contact radius is $a=\lambda_{F} / 4 \pi$.

of Eq. (20) describes the conductance $G(V)=G_{c}(V)$ in the absence of a defect $(g=0)$.

For a contact of small diameter $a \ll \lambda_{F}$, Eq. (20) can be simplified and the conductance is given by

$$
\begin{aligned}
G(V)= & G_{c}(V)\left\{1-\frac{6 \pi m^{*} g}{\hbar^{2}} \frac{A^{2}\left(\tilde{k}_{F} \gamma\right)}{\tilde{k}_{F} \gamma^{4}} z_{0}^{2}\left(1+\frac{6 m^{*} e V}{\hbar^{2} \widetilde{k}_{F}^{2}}\right)\right. \\
& \left.-\frac{72 \pi g m^{*}}{\hbar^{2} \widetilde{k}_{F}^{7}} \frac{z_{0}^{2}}{\gamma^{4}} \int_{k_{F}}^{\tilde{k}_{F}} d k k^{4} A^{2}(k \gamma)\right\},
\end{aligned}
$$

where $\gamma=\sqrt{z_{0}^{2}+\left|\rho_{0}\right|^{2}}$ is the distance between the contact and the defect. The conductance $G_{c}(V)$ of the tunnel junction of small cross section $S=\pi a^{2} \ll \lambda_{F}^{2}$ is given by

$$
G_{c}(V)=\frac{4 \pi e^{2} \varepsilon_{F}^{2} \widetilde{k}_{F}^{2} a^{4}}{9 \hbar U^{2}}=\frac{\pi^{2}}{9} T_{b}\left(\widetilde{k}_{F}\right) \frac{2 e^{2}}{h}\left(\widetilde{k}_{F} a\right)^{4},
$$

for small transmission coefficient $T_{b}(k)=\hbar^{4} k^{2} / m^{* 2} U^{2} \ll 1$.

In numerical calculations we use a value for the dimensionless parameter $2 \pi m^{*} g k_{F} / \hbar^{2}=0.3$ to characterize the strength of the defect scattering. Figure 3 shows a plot of the dependence of the normalized conductance $G(0) / G_{c}(0)$, Eq. (20), for the contact as a function of the position of the defect $\left(\rho_{0}, z_{0}\right)$ in the limit of low voltage $V \rightarrow 0$. We observe a suppression of the conductance that is largest when the contact is placed directly above the defect and find that $G$ is an oscillatory function of the defect position. In Fig. 4 we show the voltage dependence of the normalized conductance $G(V) / G_{c}(V)$, Eq. (20), for $\rho_{0}=0$ and as a function of the depth $z_{0}$ of the defect under the metal surface.

\section{BALLISTIC CONTACT}

In this section we consider another limit of a junction, a cylindrically symmetric, ballistic contact of adiabatic shape

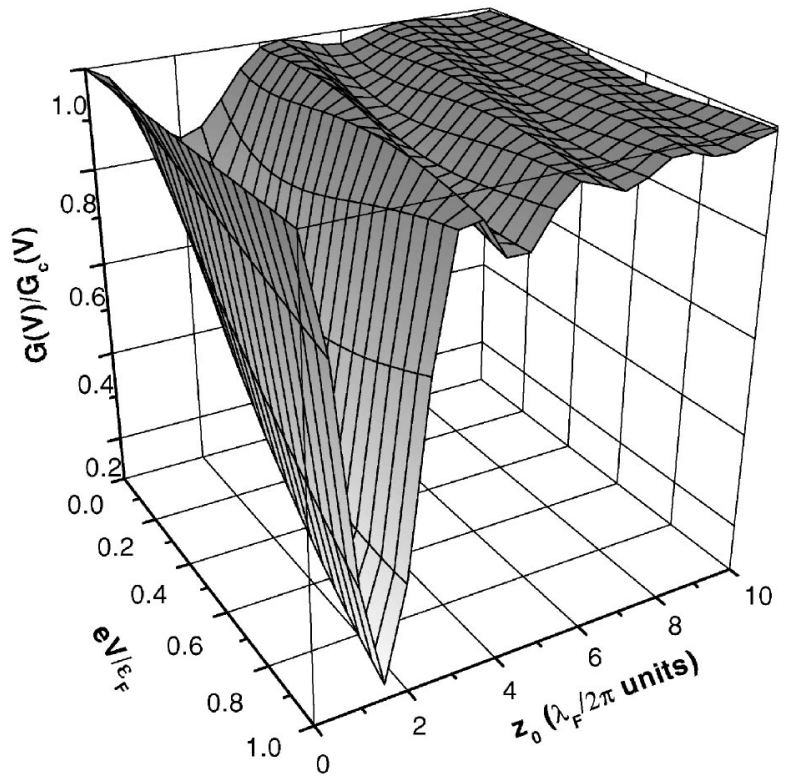

FIG. 4. Voltage dependence of the normalized conductance $G(V) / G_{c}(V)$ of a tunnel point contact for $\rho_{0}=0$ and as a function of the depth $z_{0}$ of the defect under metal surface, contact radius is $a=\lambda_{F} / 4 \pi$.

(Fig. 5). The center of the contact is characterized by a $\delta$-function potential barrier of amplitude $U$. In one of the banks of the contact a single defect is situated at the point $\mathbf{r}_{0}=\left(\rho_{0}, z_{0}\right)$, in the half-space $z_{0}>0$, such that the distance $\gamma$ between the center of the contact $\mathbf{r}=0$ and the defect is much larger than the characteristic length $L$ of the constriction (see Fig. 5). The shape of the contact is described by the radius as a function of the $z$ coordinate $a(z)$. The contact size is given by $a(0)=a_{0}$, while $a(z) \rightarrow \infty$ for $|z| \rightarrow \infty$. The adiabatic condition implies that the radius of the contact $a(z)$ varies slowly on the scale of the Fermi wavelength. As a result, the electric potential $V(\mathbf{r})$ drops dominantly over the same characteristic length $L$, as can be derived from the condition of electroneutrality. In the Landauer formalism the exact distribution of $V(\mathbf{r})$ is not important for determining the conductance of a quantum constriction, which can be expressed using only the difference of potentials $V$ in the banks far from the contact. We will consider the effect of quantum interference on the conductance under conditions $e V / \varepsilon_{F} \ll 1$ and $k_{F} \gamma\left(e V / \varepsilon_{F}\right)>1$. Fluctuations of $G(V)$ results from the phase shift $\Delta \varphi$ that the wave function accumulates after be-

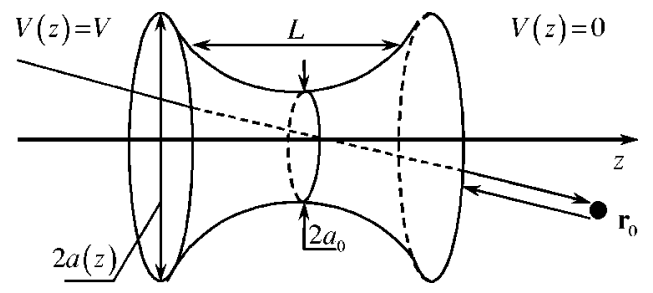

FIG. 5. Model of a ballistic contact of adiabatic shape having a defect sitting nearby. The trajectories of electrons that are transmitted through the contact and reflected from the defect are shown schematically. 
ing scattered by the defect and reflected by the contact, $\Delta \varphi \sim\left(e V / \varepsilon_{F}\right) k_{F} \gamma$. If $\gamma \gg L$, the main part of the electron trajectory is situated in the region where the local electric potential $V(\mathbf{r})$ differs only little from its value $V=0$ in the bank at $z \rightarrow \infty$, and we neglect this small variation of the potential. Assuming hard wall boundary conditions, we need to solve the Schrödinger equation,

$$
\nabla^{2} \psi_{\alpha}(\rho, z)+\frac{2 m^{*}}{\hbar^{2}}\left[\varepsilon-g \delta\left(\mathbf{r}-\mathbf{r}_{0}\right)-U \delta(z)\right] \psi_{\alpha}(\rho, z)=0,
$$

with the boundary conditions

$$
\psi_{\alpha}(|\rho|=a(z) ; z)=0,
$$

and $\alpha$ represents the full set of quantum numbers.

In the adiabatic approximation ${ }^{15,16}$ the "fast" transverse and "slow" longitudinal variables in Eq. (23) can be separated and the wave function takes on the form

$$
\psi_{\alpha}(\rho, z)=\psi_{\perp \beta}(\rho, z) \varphi_{\beta \varepsilon}(z),
$$

where $\beta=(m, n)$ is a set of two discrete quantum numbers, ${ }^{17}$ which define the transverse local eigenvalues $\varepsilon_{\beta}(z)$ and eigenfunctions $\psi_{\perp \beta}(\rho, z)$. The function $\psi_{\perp \beta}(\rho, z)$ depends on the coordinate $z$ as a local parameter, and its derivatives with respect to $z$ are small. Therefore Eq. (23) can be separated into two equations,

$$
\begin{gathered}
\nabla_{\rho}^{2} \psi_{\perp \beta}(\rho)=\frac{2 m^{*}}{\hbar^{2}} \varepsilon_{\beta}(a), \\
\frac{d^{2} \varphi_{\beta \varepsilon}}{d z^{2}}+\frac{2 m^{*}}{\hbar^{2}}\left[\varepsilon-\varepsilon_{\beta}(a)\right]=0 .
\end{gathered}
$$

The functions $\psi_{\perp \beta}(\rho)$ and $\varphi_{\beta \varepsilon}(z)$ satisfy the following conditions:

$$
\begin{gathered}
\psi_{\perp \beta}(|\rho|=a)=0 \\
\left.\frac{d \varphi_{\beta \varepsilon}(z)}{d z}\right|_{z_{0}+0}-\left.\frac{d \varphi_{\beta \varepsilon}(z)}{d z}\right|_{z_{0}-0}=\frac{2 m^{*} g}{\hbar^{2}}\left|\psi_{\perp \beta}\left(\rho_{0}\right)\right|^{2} \varphi_{\beta \varepsilon}\left(z_{0}\right) \\
\varphi_{\beta \varepsilon}^{i n c}(z) \rightarrow e^{i k z} \text { for } z \rightarrow-\infty \\
\left.\frac{d \varphi_{\beta \varepsilon}(z)}{d z}\right|_{+0}-\left.\frac{d \varphi_{\beta \varepsilon}(z)}{d z}\right|_{-0}=\frac{2 m^{*} U}{\hbar^{2}} \varphi_{\beta \varepsilon}(0)
\end{gathered}
$$

where $k=\sqrt{2 m^{*} \varepsilon} / \hbar$ and $\varphi_{\beta \varepsilon}^{i n c}(z)$ is the incident wave. Condition (30) means that we consider a wave $\varphi_{\beta \varepsilon}^{i n c}(z)$ of unit amplitude, which moves from $-\infty$ toward the contact.

For the subsequent calculations we make the explicit choice for the shape of the contact $a(z)=a_{0} \cosh (z / L)$. The condition of adiabaticity for this dependence $a(z)$ is $L \gg \lambda_{F}$. The solution of Eq. (26) is given by

$$
\psi_{\perp \beta}(\rho, \varphi, z)=\frac{1}{\sqrt{\pi} a(z) J_{m+1}\left(\gamma_{m n}\right)} J_{m}\left(\gamma_{m n} \frac{\rho}{a(z)}\right) e^{i m \varphi},
$$

having eigenvalues

$$
\varepsilon_{\beta}=\frac{\hbar^{2} \gamma_{m n}^{2}}{2 m^{*} a^{2}(z)}, \quad n=0,1,2, \ldots, \quad m=0, \pm 1, \pm 2, \ldots
$$

Here, we use cylindrical coordinates $\rho=(\rho, \varphi, z)$ and $\gamma_{m n}$ is the $n$th zero of Bessel function $J_{m}$. The energy spectrum (33) describes the quantized energy levels inside the constriction $(z<L)$ and a quasicontinuous spectrum at $z \gg L$ (the distance between the levels $\Delta \varepsilon_{\beta} \rightarrow 0$ at $|z| \rightarrow \infty$ ).

First, we consider a contact without an explicit barrier $(U=0)$. A general solution for the longitudinal wave function $\varphi_{\beta \varepsilon}(z)$ in Eq. (27) has the form,

$$
\begin{aligned}
\varphi_{\beta \varepsilon}(z)= & A\left(1-\xi^{2}\right)^{-(i k L / 2)} \\
& \times F\left(-i k L-s,-i k L+s+1,-i k L+s, \frac{1-\xi}{2}\right)+B(1 \\
& \left.-\xi^{2}\right)^{-(i k L / 2)}\left(\frac{1-\xi}{2}\right)^{i k L} F\left(-s ; s+1,1+i k L, \frac{1-\xi}{2}\right),
\end{aligned}
$$

where $F(a, b, c ; \xi)$ is the hypergeometric function, $\xi=\tanh (z / L)$, and $s=\frac{1}{2}\left(-1+i \sqrt{\left(2 L \gamma_{m n} / a_{0}\right)^{2}-1}\right)$. The constants $A$ and $B$ can be found from the conditions (29) and (30). By using the asymptotic form of hypergeometric function at $z>z_{0} \gg L$ and in the limit of a small electron-impurity interaction constant $m^{*} g k_{F} / \hbar^{2} \ll 1$, we find

$$
\varphi_{\beta \varepsilon}(z)=t_{\beta}\left[1+\frac{g m^{*}}{i k \hbar^{2}}\left|\psi_{\perp \beta}\left(\rho_{0} ; z_{0}\right)\right|^{2}\left(1+r_{\beta} e^{i k z_{0}}\right)\right] e^{i k z},
$$

where $r_{\beta}$ and $t_{\beta}$ are the amplitudes of the reflected and transmitted waves far from the contact

$$
\begin{gathered}
r_{\beta}=\frac{\Gamma(-i k L-s) \Gamma(-i k L+s+1) \Gamma(i k L)}{\Gamma(-s) \Gamma(s+1) \Gamma(-i k L)}, \\
t_{\beta}=\frac{\Gamma(-i k L-s) \Gamma(-i k L+s+1)}{\Gamma(-i k L) \Gamma(1-i k L)} .
\end{gathered}
$$

The expression for the current takes the form

$$
\begin{aligned}
I(V)= & \frac{e}{\pi \sqrt{2 m^{*}}} \sum_{\beta} \int_{z \gg L} d \mathbf{S} \int \frac{d \varepsilon}{\sqrt{\varepsilon}} \operatorname{Im}\left(\psi_{\alpha}^{*} \nabla \psi_{\alpha}\right)\left[n_{F}(\varepsilon)-n_{F}(\varepsilon\right. \\
& +e V)] .
\end{aligned}
$$

Substituting the wave function $\psi_{\alpha}(\rho, z)$ from (25) into Eq. (38), with $\psi_{\perp \beta}(\rho, z)$ given by (32) and $\varphi_{\beta \varepsilon}(z)$ by (35), and carrying out the integration over $\rho \leqslant a(z)$ at $z>z_{0}$, we find the total current trough is the contact for $k L \gg 1$ and $L \gg a_{0}$, 


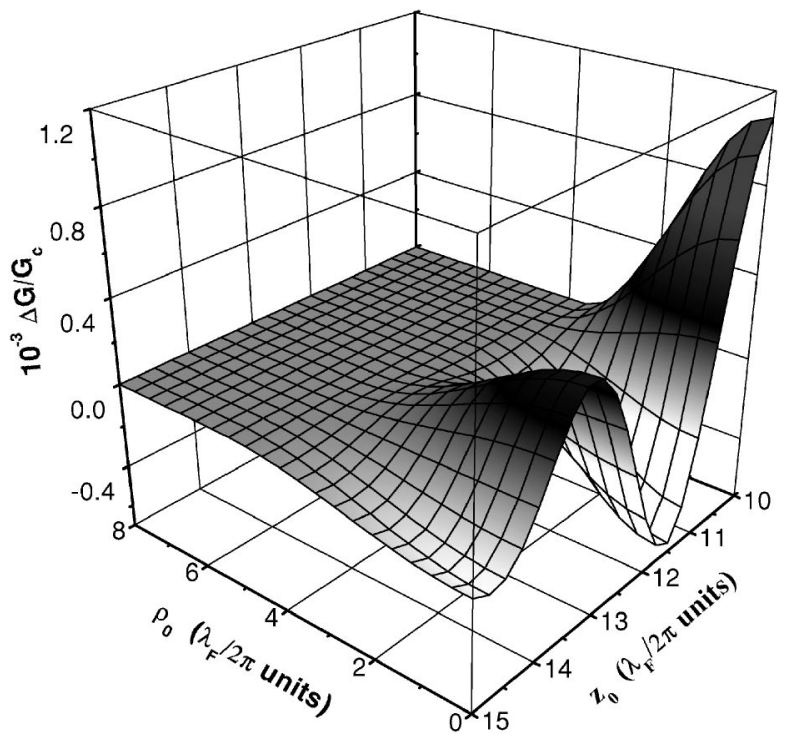

FIG. 6. Dependence of the oscillatory part of normalized conductance $\Delta G(0) / G_{c}(0)$ of the ballistic point contact with the position coordinates of the defect $\left(\rho_{0}, z_{0}\right)$.

$$
\begin{aligned}
I(V)= & \frac{2 e}{h} \int d \varepsilon \sum_{\beta} T_{\beta}\left[n_{F}(\varepsilon)-n_{F}(\varepsilon+e V)\right] \\
& \times\left\{1+\frac{g m^{*}}{\hbar^{2} k}\left|\psi_{\perp \beta}\left(\rho_{0}\right)\right|^{2}\left|r_{\beta}\right| \cos \left(2 k z_{0}+\varphi_{\beta}\right)\right\} .
\end{aligned}
$$

Here,

$$
\begin{gathered}
T_{\beta}(\varepsilon)=\frac{1}{1+\exp \left[2 \pi L\left(k_{\beta}-k\right)\right]}, \\
\left|r_{\beta}(\varepsilon)\right|=\frac{1}{\sqrt{\exp \left[2 \pi L\left(k-k_{\beta}\right)\right]+1}}, \\
\varphi_{\beta}(\varepsilon)=\left(k+k_{\beta}\right) L\left\{1-\ln \left[\left(k+k_{\beta}\right) L\right]\right\}-\left(k-k_{\beta}\right) L \psi\left(\frac{1}{2}\right)-2 k L(1 \\
-\ln k L),
\end{gathered}
$$

$k_{\beta}=\sqrt{2 m^{*} \varepsilon_{\beta}(0)} / \hbar$ is the quantized momentum of the transverse electron motion at the center of the contact, $\psi\left(\frac{1}{2}\right)=\Gamma^{\prime}\left(\frac{1}{2}\right) / \Gamma\left(\frac{1}{2}\right) \approx-1.96$.

The conductance $G(V)=d I / d V$, in the low-temperature limit, is given by

$$
\begin{aligned}
G(V)= & G_{0} \sum_{\beta} T_{\beta}\left(\varepsilon_{F}\right) \\
& \times\left\{1+\frac{g m^{*}}{\hbar^{2} k_{F}}\left|\psi_{\perp \beta}\left(\rho_{0}\right)\right|^{2}\left|r_{\beta}\right| \cos \left(2 \widetilde{k}_{F} z_{0}+\varphi_{\beta}\right)\right\},
\end{aligned}
$$

where $G_{0}=2 e^{2} / h$ is the quantum of conductance and $\tilde{k}_{F}=\sqrt{k_{F}^{2}+2 m e V / \hbar^{2}}$. All energy-dependent functions are taken at $\varepsilon=\varepsilon_{F}$. We also used the condition $e V / \varepsilon_{F} \ll 1$.

The transmission coefficient is exponentially small $T_{\beta}(\varepsilon) \sim \exp \left[-2 \pi L\left(k_{\beta}-k\right)\right]$ for $\varepsilon \ll \varepsilon_{\beta}(0)$, while $T_{\beta}(\varepsilon) \rightarrow 1$ above this energy, $\varepsilon \gg \varepsilon_{\beta}(0)$. For $\left|\varepsilon-\varepsilon_{\beta}(0)\right| \ll \varepsilon_{\beta}(0)$, Eq. (40)

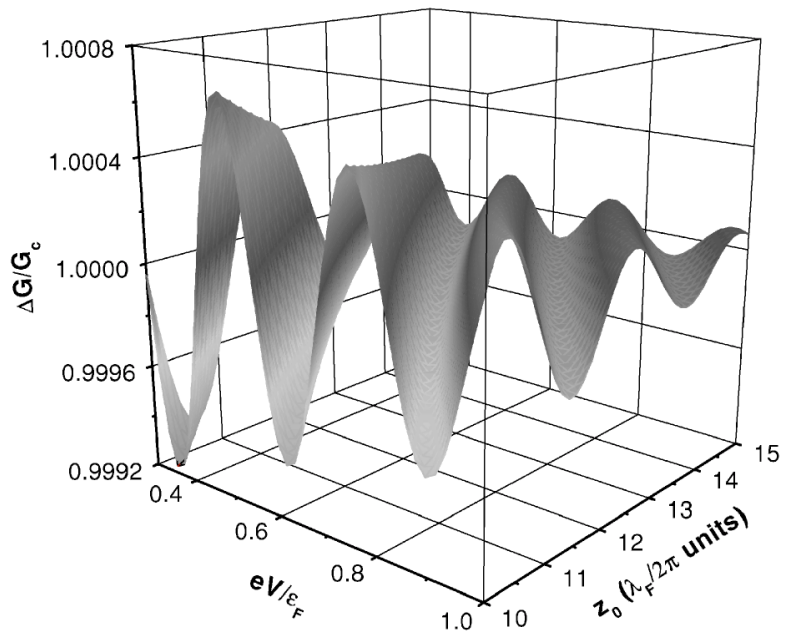

FIG. 7. Voltage dependence of the oscillatory part of normalized conductance $\Delta G(V) / G_{c}(V)$ for an adiabatic ballistic point contact for $\rho_{0}=0$ as a function of the depth $z_{0}$ of the defect under metal surface.

agrees with the formula for the transmission coefficient that can be obtained in such a case for an arbitrary dependence $a(z)$, by using an expansion near the point of minimum cross section, $a(0)$ (see, e.g., Ref. 18). For very long constrictions $(L \rightarrow \infty)$, Eq. (40) transforms to a step function $\Theta\left(\varepsilon-\varepsilon_{\beta}(0)\right)$. For large $L$ the electrons are strongly reflected by the contact when $k \simeq k_{\beta}$. Hence, for observation of conductance oscillations in an adiabatic ballistic constriction the contact diameter should be chosen in such a way that $\varepsilon_{F} \gtrsim \varepsilon_{\beta}(0)$, i.e., not very far from the middle of a conductance step.

In the case $U \neq 0$ the boundary condition (31) must be taken into account. At $k \gg k_{\beta}$, reflection due to the shape of the contact is negligibly small, as discussed above, and the conductance $G(V)$ is also described by Eq. (43), but with

$$
\begin{gathered}
T_{\beta}(\varepsilon)=\frac{1}{1+\left(m^{*} U / \hbar^{2} k_{\beta}^{\prime}\right)^{2}}, \\
\left|r_{\beta}(\varepsilon)\right|=\frac{m^{*} U / \hbar^{2} k_{\beta}^{\prime}}{\sqrt{1+\left(m^{*} U / \hbar^{2} k_{\beta}^{\prime}\right)^{2}}}, \\
\varphi_{\beta}(\varepsilon)=\arcsin \left(\frac{1}{\sqrt{1+\left(m^{*} U / \hbar^{2} k_{\beta}^{\prime}\right)^{2}}}\right),
\end{gathered}
$$

where $k_{\beta}^{\prime}=\sqrt{k^{2}-k_{\beta}^{2}}$.

Figure 6 shows the dependence of the oscillatory part of conductance $\Delta G(V)=G(V)-G_{c}(V)$, Eq. (43), on the position of the defect at low voltage $(V \rightarrow 0)$ for a contact without barrier $(U=0)$. Here, $G_{c}=G_{0} \Sigma_{\beta} T_{\beta}$ is the conductance in the absence of a defect $(g=0)$. Figure 7 shows the dependence of the $\Delta G(V)$ on applied bias voltage for a defect sitting on the axis of the contact $\left(\rho_{0}=0\right)$ and as a function of the distance $z_{0}$ from the contact center. In creating the plots of Figs. 6 and 7 we have used dimensionless parameter $2 \pi m^{*} g k_{F} / \hbar^{2}=0.5$, $2 \pi a_{0}=2.405 \lambda_{F}$, and $2 \pi L=10 \lambda_{F}$, corresponding to a contact having one allowed quantum conductance mode. 


\section{DISCUSSION}

The presence of an elastic scattering center located inside the bulk, either in the vicinity of a tunnel contact in an STM configuration or in one of the banks of a ballistic point contact, has been shown to cause oscillatory fluctuations in the conductance of the junction. For small contact radii $\left(a \ll \lambda_{F}\right)$, these oscillations result solely from interference of electron waves that are directly transmitted on the one hand, and electrons that are both backscattered by the defect and again reflected by the contact on the other. What now follows is a discussion as to whether this effect can be employed experimentally for three-dimensional mapping of subsurface impurities.

In the case of a tunnel contact, the oscillatory part of the conductance can be expressed by

$$
\frac{G^{o s c}(V)}{G_{c}} \propto \frac{z_{0}^{2} \lambda_{F}^{2}}{\gamma^{4}} \sin 2 \tilde{k}_{F} \gamma, \quad \tilde{k}_{F} \gamma \gg 1,
$$

where $\tilde{k}_{F}=\sqrt{k_{F}^{2}+2 m e V / \hbar^{2}}$ is the wave vector of electrons that are passing through the orifice, $z_{0}$ is the depth of the defect under the surface, and $\gamma$ is the distance between the contact and the defect. Comparing this to the results found for a ballistic contact, where

$$
\frac{G^{o s c}(V)}{G_{c}} \propto \frac{\lambda_{F}^{2}}{a^{2}\left(z_{0}\right)} \cos \left(2 \widetilde{k}_{F} z_{0}+\varphi_{\beta}\right)
$$

(here $\varphi_{\beta}$ is the phase the electron acquires after reflection by the contact), we see that although both oscillations have similar arguments, the expression for the ballistic case has an extra phase $\varphi_{\beta}$, which depends nonlinearly on the wave vector $k$, making the signal hard to identify. Furthermore, the adiabatic condition, being an essential assumption in the ballistic model, cannot be readily achieved experimentally.

Therefore, choosing the tunnel contact for experimental application seems most sensible. In that situation we can expect the information in the conductance signal about a defect's whereabouts to be twofold: the amplitude will decrease with growing distance $\gamma$, whereas the frequency of the oscillation is expected to increase upon enlarging the distance from contact to defect. The actual experiment would consist of sensitively measuring $(d I / d V)(V)$ curves on a tight grid of $\rho$ coordinates. The lateral positions of defects could then be identified as the centers of radially symmetric patterns in this signal. Next, the depth of an impurity should be derived from the period of the oscillation in the $(d I / d V)(V)$ curve at $\rho_{0}$.

Assuming the numerical parameter $2 \pi m^{*} g k_{F} / \hbar^{2}=0.5$ introduced in Sec. III (which can be shown to be applicable for hard wall scatterers with atomic radius) and choosing the orifice to be located exactly above the defect $\left(\gamma=z_{0}\right)$, the amplitude of the oscillation is expected to be $10^{-1} G_{c}$ for $z_{0}=3 \mathrm{~nm}$ (with $k_{F}=10^{10} \mathrm{~m}^{-1}$ ).

Note that the chosen value of the interaction constant is rather large. We use such a value of the parameter to show the investigated effects more clearly in illustrations. For real values of parameter $g \approx 10^{-35} \mathrm{erg} \mathrm{cm}^{3}$, which can be estimated from an electron effective scattering cross section $\sim 1 \AA^{2}$, the relative amplitude of oscillations is $10^{-2} / 10^{-3} G_{c}$.

Comparing this to previous STS experiments, ${ }^{19}$ where signal-to-noise ratios of $5 \times 10^{-4}$ (at $1 \mathrm{nA}, 400 \mathrm{~Hz}$ sample frequency) have been achieved, we should be able to measure defects located more than ten atomic layers under the surface.

As the period of the oscillation becomes longer for small $z_{0}$, the minimum discernable depth will be determined by the maximum voltage that can be applied over the junction. For example, $30 \mathrm{mV}$ is sufficient for probing a quarter of a conductance oscillation caused by a defect at $1 \mathrm{~nm}$ depth. The increase of the noise level inherent to measuring at elevated voltages will not pose a problem because the amplitude of the signal is much higher for small depths.

Finally, the anisotropy of the electronic structure will have to be taken into account. Materials with an almost spherical Fermi surface, such as $\mathrm{Al}$ or $\mathrm{Au}$, realizing the condition of a free-electron gas, are expected to be most suitable. Furthermore, deviations of spherical symmetry might be used as a secondary proof for the effectiveness of the method, i.e., in the case of $\mathrm{Au}(111)$, where the "necks" in the Fermi surface should cause a defect to be invisible when probed exactly from above.

This research was supported partly by the program "Nanosystems, nanomaterials, and nanotechnology" of National Academy of Sciences of Ukraine. Ye. S. A. wishes to acknowledge the INTAS grant for Young Scientists.
${ }^{1} \mathrm{Ph}$. Ebert, M. Heinrich, M. Simon, C. Domke, K. Urban, C. K. Shih, M. B. Webb, and M. G. Lagally, Phys. Rev. B 53, 4580 (1996).

${ }^{2}$ S. Heinze, R. Abt, S. Blügel, G. Gilarowski, and H. Niehus, Phys. Rev. Lett. 83, 4808 (1999).

${ }^{3}$ M. Schmid, W. Hebenstreit, P. Varga, and S. Crampin, Phys. Rev. Lett. 76, 2298 (1996).

${ }^{4}$ Hongbin Yu, C. S. Jiang, Ph. Ebert, and C. K. Shih, Appl. Phys. Lett. 81, 2005 (2002).

${ }^{5}$ M. F. Crommie, C. P. Lutz, and D. M. Eigler, Nature (London) 363, 524 (1993); Science 262, 218 (1993).
${ }^{6}$ S. Crampin, J. Phys.: Condens. Matter 6, L613 (1994).

${ }^{7}$ C. Untiedt, G. Rubio Bollinger, S. Vieira, and N. Agraït, Phys. Rev. B 62, 9962 (2000).

${ }^{8}$ B. Ludoph and J. M. van Ruitenbeek, Phys. Rev. B 61, 2273 (2000).

${ }^{9}$ A. Halbritter, Sz. Csonka, G. Mihály, O. I. Shklyarevskii, S. Speller, and H. van Kempen, Phys. Rev. B 69, 121411 (2004).

${ }^{10}$ I. F. Itskovich and R. I. Shekhter, Fiz. Nizk. Temp. 11, 373 (1985) [Sov. J. Low Temp. Phys. 11, 202 (1985)].

${ }^{11}$ A. Namiranian, Yu. A. Kolesnichenko, and A. N. Omelyanchouk, Phys. Rev. B 61, 16796 (2000). 
${ }^{12}$ Ye. S. Avotina and Yu. A. Kolesnichenko, Fiz. Nizk. Temp. 30, 209 (2004) [J. Low Temp. Phys. 30, 153 (2004)].

${ }^{13}$ Ye. S. Avotina, A. Namiranian, and Yu. A. Kolesnichenko, Phys. Rev. B 70, 075308 (2004).

${ }^{14}$ I. O. Kulik, Yu. N. Mitsai, and A. N. Omelyanchouk, Zh. Eksp. Teor. Fiz. 63, 1051 (1974).

${ }^{15}$ L. I. Glazman, G. B. Lesovik, D. E. Khmel'nitskii, and R. I. Shekhter, JETP Lett. 48, 238 (1988).
${ }^{16}$ E. N. Bogachek, A. M. Zagoskin, and I. O. Kulik, Sov. J. Low Temp. Phys. 16, 796 (1990).

${ }^{17}$ L. D. Landau and E. M. Lifshits, Quantum Mechanics (Pergamon, Oxford, 1977).

${ }^{18}$ E. N. Bogachek, A. G. Scherbakov, and Uzi Landman, Phys. Rev. B 54, R11094 (1996).

${ }^{19}$ B. C. Stipe, M. A. Rezaei, and W. Ho, Rev. Sci. Instrum. 70, 137 (1999). 